Cahiers de philosophie de l'université de

\title{
Imitation et émotion : Bergson lecteur de Tarde
}

\section{Arnaud Bouaniche}

\section{CpenEdition}

Journals

Édition électronique

URL : https://journals.openedition.org/cpuc/313

DOI : $10.4000 /$ cpuc.313

ISSN : 2677-6529

\section{Éditeur}

Presses universitaires de Caen

\section{Édition imprimée}

Date de publication : 31 décembre 2017

Pagination : 59-72

ISBN : 978-2-84133-859-7

ISSN : 1282-6545

Référence électronique

Arnaud Bouaniche, «Imitation et émotion : Bergson lecteur de Tarde», Cahiers de philosophie de I'université de Caen [En ligne], 54 | 2017, mis en ligne le 01 février 2019, consulté le 03 février 2023 URL : http://journals.openedition.org/cpuc/313 ; DOI : https://doi.org/10.4000/cpuc.313

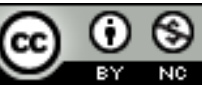

Creative Commons - Attribution - Pas d'Utilisation Commerciale 4.0 International - CC BY-NC 4.0 https://creativecommons.org/licenses/by-nc/4.0/ 


\section{Imitation et émotion : Bergson lecteur de Tarde}

$\mathrm{L}$

E BUT DE CETTE ÉTUDE n'est pas de proposer une confrontation d'ensemble des œuvres de Bergson et de $\operatorname{Tarde}^{1}$, mais plus modestement d'envisager leur relation sur un point précis et limité où ils paraissent se croiser, au sens où deux personnes peuvent le faire: sans venir du même endroit, ni aller dans la même direction.

Un point de recoupement remarquable - parmi de nombreux autres possibles - entre les deux penseurs réside selon nous dans l'attention et l'importance extrêmes qu'ils accordent l'un et l'autre dans leur œuvre à l'influence exercée, dans la société ou dans l'histoire, par certains individus exceptionnels, innovateurs ou créateurs, sur d'autres individus, imitateurs ou continuateurs. On pourrait appeler cette influence la causalité de l'exemple $^{2}$. Certes, Bergson et Tarde pensent différemment cette influence, comme «émotion " pour le premier, comme «imitation " pour le second, et il y a ici entre eux, bien entendu, beaucoup plus qu'une simple différence terminologique, à tel point qu'il n'est même pas certain que l'on trouve derrière ces deux termes exactement le même phénomène. Toutefois, ce qui demeure frappant dans un premier temps, c'est, d'une part, la reconnaissance par Bergson lui-même d'une affinité entre l'émotion morale et le "désir de ressembler ${ }^{3}$ (qui se traduit par l'adoption pratique d'un modèle),

1. Une telle étude reste selon nous à conduire. Pour une confrontation entre Tarde et Bergson, on trouvera cependant des éléments nombreux et précis dans l'important ouvrage de J. Milet, Gabriel Tarde et la philosophie de l'histoire, Paris, J. Vrin, 1970, notamment p. 131-135 et p. 386-391.

2. Sur ce point, et dans une perspective plus large, voir notre étude: A. Bouaniche, «Bergson et la causalité de l'exemple à la lumière des sciences sociales et biologiques: charisme, imitation, émotion », Implications philosophiques, janvier 2014, dossier thématique «Bergson ou la science», p. 90-113.

3. Il n'est pas certain que l'imitation soit volontaire chez Tarde, mais pas plus que chez Bergson du reste. Ce dernier précise en effet que l'émotion que j'éprouve en présence d'une conduite ou d'une parole exemplaires suscite en moi «une inclination à laquelle je ne voudrais pas 
et, d'autre part, la grande proximité des démarches de Tarde et de Bergson, puisqu'à travers l'imitation et l'émotion, chacun accorde à un concept d'origine psychologique une portée profonde, et un sens métaphysique. À la pointe de la réalité, historique ou sociale, où elles émergent et circulent entre les hommes, l'imitation et l'émotion se voient en effet attribuer une valeur de sommet, comme deux manifestations de l'expansion et du triomphe de ce qui est à leurs yeux au principe du réel: «désir» et «croyance» selon Tarde, «durée» selon Bergson. Or, c'est peut-être en lisant Tarde, qui le précède sur ce point, que Bergson a puisé une inspiration déterminante pour envisager et penser la nature dynamique du lien qui, dans son dernier livre, Les deux sources de la morale et de la religion (1932), entraîne les hommes sur les voies d'une transformation morale dans l'histoire.

\section{Une action «d'esprit à esprit »}

Il convient d'emblée de le rappeler: Bergson est bien lecteur de Tarde - et réciproquement d'ailleurs, au moins en ce qui concerne l'Essai sur les données immédiates de la conscience (1889), que Tarde cite très favorablement ${ }^{4}$. C'est sans doute en 1904 que Bergson est conduit à lire de plus près l'œuvre du sociologue, dans une période où le hasard a voulu qu'il lui succède au Collège de France, à la fin de cette même année, pour occuper la chaire de philosophie moderne. Mais si la part de circonstance est réelle dans cette lecture, rien n'imposait à Bergson de s'enthousiasmer. Or tel est bien le cas, puisque c'est un hommage appuyé, vibrant même, que celui-ci adresse à Tarde, dans trois textes brefs et importants ${ }^{5}$ : l'un est un discours prononcé en 1909 lors de l'inauguration d'un monument dédié à sa mémoire, l'autre est la préface d'une anthologie de morceaux choisis de l'œuvre du sociologue ${ }^{6}$ parue la même année. Le troisième est antérieur, et inédit. Il s'agit d'un passage issu d'un cours de Bergson donné au Collège de France sur «L'évolution du problème de la liberté», daté du 6 décembre 19047. Bergson y salue le génie de Tarde, qui vient de mourir quelques mois plus tôt (en mai), tout en exprimant son désir

résister ", si justement je devais prendre conscience de cette nécessité (H. Bergson, Les deux sources de la morale et de la religion, Paris, PUF (Quadrige), 2008, p. 45).

4. Voir G. Tarde, Les lois de l'imitation, Paris, Seuil (Les empêcheurs de penser en rond Euvres de Gabriel Tarde, 2-1), 2001, chap. V, p. 204, note 1.

5. H. Bergson, Écrits philosophiques, Paris, PUF (Quadrige), 2011, p. 375 sq.

6. Ibid., p. 377 sq.

7. Ce cours est à paraître aux Presses universitaires de France, sous la responsabilité éditoriale d'Arnaud François. Nous le remercions d'avoir porté à notre connaissance ce passage et de nous l'avoir transmis; nous le reproduisons en annexe à la fin de cette étude. 
de lui consacrer un cours, qu'il ne fera finalement jamais, mais les deux textes de 1909 confirmeront l'intérêt réel de Bergson pour Tarde, tout en témoignant d'une lecture approfondie des Lois de l'imitation au moins. Si ce passage de 1904 est toutefois précieux, c'est en raison du rapprochement que Bergson y esquisse entre Tarde et Spencer, qu'il présente comme deux philosophes opposés et complémentaires (le «déductif» et l'«intuitif»), conférant du même coup à Tarde une envergure inattendue et remarquable quand on connaît l'importance au contraire manifeste de Spencer dans son œuvre ${ }^{8}$.

C'est cependant la préface de 1909 qui apparaît comme décisive pour notre propos. Bergson y insiste particulièrement sur le thème d'une causalité spécifique, du modèle ou de l'exemple sur ceux qui l'imitent, véritable action exercée par un esprit sur un autre esprit:

Ce qui domine dans toute sa philosophie, c'est un certain point de vue très original sur la causalité. La causalité par excellence est, à ses yeux, celle qui opère dans les sociétés humaines, où un individu invente et où d'autres individus l'imitent. Elle ne se ramène à aucun des types de causalité décrits par les physiciens et les métaphysiciens. L'imitation est une certaine action sui generis qui s'exerce d'esprit à esprit' .

Pour comprendre l'intérêt que suscite chez Bergson cette conception d'une causalité psycho-sociale entre inventeurs et imitateurs, il faut revenir à la situation chronologique de ce passage. Nous sommes au lendemain de L'évolution créatrice. Dans ce grand livre daté de 1907, la vie, conçue à l'échelle cosmologique comme un "élan " (le fameux «élan vital»), atteste dans l'univers, selon Bergson, un certain triomphe - qui atteint avec l'homme son degré le plus haut -, triomphe de l'action et de la liberté sur la passivité et l'inertie de la matière. Mais bien vite, ce sont les limites de cette victoire qui apparaissent à Bergson, autrement dit, la menace d'une fatigue, d'une exténuation, d'une retombée de cette poussée conquérante et créatrice, qui bientôt s'inverse et s'enroule sur elle-même en une tendance conservatrice qui finit par ne plus viser que sa propre reproduction, sous les traits de «l'espèce» d'abord, de la «société » ensuite, de sorte que c'est la destination de la vie humaine, autrement dit sa dimension créatrice, qui paraît sur le point d'être compromise. Or, la fonction de certains

8. Il convient d'ajouter que l'œuvre de Spencer n'est pas non plus sans importance dans celle de Tarde. Ce dernier a également lu Spencer et, comme Bergson, même si c'est dans une perspective différente, il critique constamment son évolutionnisme; sur ce point, voir J. Milet, Gabriel Tarde et la philosophie..., p. 244 sq.

9. H. Bergson, Écrits philosophiques, p. 378-379. 
individus - les grandes personnalités morales dans l'histoire - paraît être selon Bergson, au point de vue cosmologique où il se place, d'assurer à l'humanité une «reprise» d'élan ou de mouvement:

La diversité des efforts [accomplis par les grands hommes de bien] se résumerait bien à quelque chose d'unique: un élan qui avait donné des sociétés closes parce qu'il ne pouvait plus entraîner la matière, mais que va ensuite chercher et reprendre, à défaut de l'espèce, telle ou telle individualité privilégiée. Cet élan se continue ainsi par l'intermédiaire de certains hommes [... ${ }^{10}$.

En accordant à l'individu ("à défaut de l'espèce») une puissance d'entraînement et de transformation, Bergson paraît opérer, comme Tarde du reste ${ }^{11}$, un retournement complet de la philosophie de l'histoire de Kant. Chez ce dernier, c'est l'espèce humaine qui peut seule accomplir les fins naturelles et culturelles de l'humanité ${ }^{12}$. Tout le problème est alors pour Bergson de comprendre comment ces individus (saints, sages, prophètes, etc.) peuvent parvenir à «entraîner» les autres hommes à leur suite. Or, c'est bien comme une «imitation » que Bergson envisage d'abord cette influence:

Pourquoi les saints ont-ils ainsi des imitateurs, et pourquoi les grands hommes de bien ont-ils entraîné derrière eux des foules? Ils ne demandent rien et pourtant ils obtiennent. Ils n'ont pas besoin d'exhorter; ils n'ont qu'à exister; leur existence est un appel ${ }^{13}$.

La seule occurrence du terme « imitateur» peut ici sembler bien vague, et à elle seule insuffisante, pour légitimer une véritable filiation avec Tarde. En revanche, la présence du verbe «entraîner ", qui semble aussitôt surgir afin d'expliciter dans la phrase l'acte dont l'imitation serait, sur un mode passif, l'envers ou l'effet, donne plus de poids à cette hypothèse. Dans un cours donné au Collège de France en 1902-1903, autour de l'œuvre de Cournot, Tarde établit en effet lui-même le lien entre les phénomènes d'imitation et d'entraînement ${ }^{14}$. À propos du cas de la propagation d'un mot nouveau dans une langue donnée, Tarde attire l'attention sur la présence chez Cournot

10. H. Bergson, Les deux sources..., p. 285.

11. Sur le rôle de l'individu dans l'histoire chez Tarde, voir J. Milet, Gabriel Tarde et la philosophie..., p. 343 sq.

12. Voir E. Kant, Idée d'une histoire universelle au point de vue cosmopolitique, prop. 1 et 2 [Ak. VIII, p. 18], in Euvres philosophiques, F. Alquié (éd.), Paris, Gallimard (Bibliothèque de la Pléiade; 317), t. II, 1985, p. 189.

13. H. Bergson, Les deux sources..., p. 30 ; nous soulignons.

14. Voir G. Tarde, Philosophie de l'histoire et science sociale: la philosophie de Cournot, Paris, Seuil (Les empêcheurs de penser en rond - Euvres de Gabriel Tarde, 2-4), 2002, p. 179 sq. 
du phénomène d' «entraînement», auquel il convient de donner son "vrai nom ", celui d' «imitation", ce que Cournot aurait fait furtivement selon Tarde, mais sans soupçonner la portée profonde d'un tel phénomène. Or, d'après Tarde, cette innovation linguistique ne doit rien à un processus impersonnel et à quelque chose comme un "génie de la langue ». Elle résulte de la propagation par imitation d'une invention sémantique individuelle, c'est-à-dire de la relation d'imitateurs à un ou plusieurs inventeurs qui ont servi de modèles et entraîné d'autres locuteurs à leur suite.

\section{L'invention morale}

Or, c'est un leitmotiv qui court dans Les deux sources de la morale et de la religion, que s'il y a des imitateurs - Bergson dit le plus souvent des «continuateurs"-, c'est parce qu'il y a d'abord des initiateurs, inventeurs ou créateurs. On comprend alors pourquoi Bergson parle à plusieurs reprises d'une «invention morale ${ }^{15}$, reconduisant par là en le reprenant jusqu'à un certain point à son compte, le couple invention-imitation, central chez Tarde. "Qui dit imitation, écrit Tarde, dit invention, innovation, par la même raison qu'une série suppose un premier terme $»^{16}$. Et Bergson: «Nous ne croyons pas à l'inconscient en histoire: les grands courants souterrains de pensée, dont on a tant parlé, sont dus à ce que des masses d'hommes ont été entraînées par un ou plusieurs d'entre eux ${ }^{17}$. À ce stade, et dans un premier temps, le point de convergence entre Tarde et Bergson paraît évident. L'un et l'autre posent à l'origine de toute nouveauté, sociale, culturelle ou historique, une invention individuelle. Soit l'émotion esthétique éprouvée par chacun d'entre nous devant la montagne, pour reprendre un exemple donné par Bergson dans le premier chapitre des Deux sources ${ }^{18}$. Cette émotion n'est pas dans les choses, mais c'est parce que Rousseau l'a éprouvée avant nous et lancée dans la circulation, explique Bergson dans une perspective ici très proche de celle de Tarde, que nous l'éprouvons après, et surtout avec lui.

On ne saurait pourtant en rester là. En effet, si Tarde maintient la référence, qu'il déclare nécessaire, à un inventeur ou à un créateur, véritable foyer d'un

15. On trouve à la lettre cette expression chez $\mathrm{G}$. Tarde, par exemple dans Les transformations $d u$ pouvoir, Paris, Seuil (Les empêcheurs de penser en rond - Euvres de Gabriel Tarde, 2-2), 2003, p. 270. Nous faisons l'hypothèse que, dans Les deux sources..., les nombreuses occurrences du mot «invention» sous la plume de H. Bergson, en alternance avec celles du mot «création», sont une trace de l'influence tardienne.

16. G. Tarde, Philosophie de l'histoire et science sociale..., p. 180.

17. H. Bergson, Les deux sources..., p. 328.

18. Ibid., p. 37-38. 
rayonnement imitatif ${ }^{19}$, il est loin de considérer que l'individualité empirique et historique de cet inventeur constitue une réalité dernière. Contrairement à Bergson, qui ne cesse de répéter qu'il y a, à l'origine de chaque création, morale ou esthétique, "quelqu'un», Tarde de son côté insiste sur le caractère seulement provisoire de cette individualité qui, lorsqu'on l'envisage dans sa genèse et son processus de constitution effectif, à un niveau infinitésimal, perd son caractère originaire et se résout aussi bien en un ensemble de flux imitatifs impersonnels de croyance et de désir, à qui l'individu remarquable sert seulement de lieu de rencontre ou de croisement, mais non pas d'élaboration. En dernière instance, «ce qui est inventé ou imité, ce qui est imité, c'est toujours une idée ou un vouloir, un jugement ou un dessein, où s'exprime une certaine dose de croyance et de désir $[\ldots] »^{20}$. Par conséquent, si Bergson a raison de voir en Tarde un allié qui fait avec lui cause commune contre un certain déterminisme alors dominant dans les sciences sociales - l'un et l'autre affirmant la part de contingence dans le devenir socio-historique -, il force assurément la position de Tarde, lorsqu'il lui attribue la thèse selon laquelle les sociétés humaines sont traversées de courants à l'origine desquels il y a une impulsion qui «vient d'un homme " ${ }^{21}$. Pour Tarde en effet, comme l'écrit Bruno Karsenti, «l'individualité [...], qu'il s'agisse d'un homme, d'un peuple ou d'une civilisation déterminée, se dissout pour apparaître sous son aspect intrinsèquement relationnel ${ }^{22}$, à tel point qu'on peut même aller jusqu'à parler chez le sociologue d'une véritable «destitution de l'individu» ${ }^{23}$. Si pourtant les pages sont innombrables dans lesquelles Tarde insiste sur l'individualité créatrice, qu'il place systématiquement à l'origine du «tout» formé par la société et résultant d'un phénomène de «suggestion-imitation », il faut comprendre que l'accent porte moins sur l'inventeur que sur l'invention, et que ce sont, en dernière instance, les «idées », les «croyances» et les « désirs» qui, à travers cette individualité, circulent et se propagent.

\section{La propagation du nouveau}

Au-delà de cette différence, c'est cependant un même problème que paraissent partager Bergson et Tarde, autour de la propagation du nouveau: comment comprendre qu'une innovation, invention ou création, suscite un écho chez d'autres, un désir de ressembler, au lieu d'être rejetée

19. Sur ce point, voir G. Tarde, Les lois sociales, Paris, Institut d'édition Sanofi-Synthélabo (Les empêcheurs de penser en rond - Euvres de Gabriel Tarde, 4), 1999, p. 56 sq.

20. G. Tarde, Les lois de l'imitation, p. 204; c'est Tarde qui souligne.

21. H. Bergson, Écrits philosophiques, p. 376.

22. B. Karsenti, «Présentation», in G. Tarde, Les lois de l'imitation, Paris, Kimé, 1993, p. XXV.

23. Ibid., p. XV. 
comme une anomalie, un écart accidentel? Pourquoi l'audace et l'initiative ouvrent-elles une «suite», au lieu d'être purement et simplement expulsées ou ignorées? Pourquoi les paroles du Christ («tendre l'autre joue», etc.), pour reprendre l'exemple que l'on trouve dans un passage central des Deux sources ${ }^{24}$, ne nous font-elles pas rire? Le mécanisme du rire, comme Bergson l'a montré ailleurs, dans un célèbre essai ${ }^{25}$, est bien en effet une tendance inscrite en l'homme par laquelle se trouvent sanctionnées toutes les infractions à l'ordre social, ce qui est par essence le cas des paroles de la «morale ouverte», dont ni le contenu ni l'origine ne relèvent de la société, mais d'une aspiration qui est toujours une création individuelle, adressée non pas à une société réellement existante, mais à la «société idéale» de l'humanité, suscitant en chacun un appel.

Ce qui est en jeu ici, c'est une causalité «sans contact» ou "à distance», d'après la conception d'une cause inédite, distincte de celle qui est ordinairement à l'œuvre dans les versions déterministes de la causalité. Bergson l'indique très furtivement dans le passage que nous citions plus haut: ce n'est pas en agissant directement sur eux, que les grands hommes de bien entraînent les autres hommes, mais sans rien leur demander, par leur simple «présence». De même lorsque Tarde s'efforce de préciser la nature de l'influence qui s'exerce entre modèle et copie, imitant et imité, dans le processus d'imitation, il évoque une «action qui consiste dans une reproduction quasi photographique d'un cliché cérébral par la plaque sensible d'un autre cerveau ${ }^{26}$. À travers l'image photographique, on comprend que l'imitation ne consiste pas en une inscription par contact direct, mais en "l'émergence d'une empreinte semblable à l'image qui progressivement apparaît sur la pellicule de film que l'on développe $»^{27}$. La logique de l'imitation telle que Tarde la conçoit est dès lors moins celle de la propagation d'un modèle qui étendrait progressivement son influence sur un ensemble d'individus donnés, que l'expression actuelle simultanée de ce modèle chez ces mêmes individus. Tarde en tire une définition du groupe social comme " collection d'êtres en tant qu'ils sont en train de s'imiter actuellement, ou en tant que, sans s'imiter actuellement, ils se ressemblent et que leurs traits communs sont des copies anciennes d'un même modèle ${ }^{28}$. La dyade imitation / invention forme ainsi l' " acte social élémentaire ${ }^{29}$, dès lors qu'il n'y a pas d'imitation sans invention.

24. H. Bergson, Les deux sources..., p. 57-58.

25. Voir H. Bergson, Le rire, Paris, PUF (Quadrige), 2008.

26. G. Tarde, Les lois de l'imitation, 2001, p. 46.

27. B. Karsenti, «Présentation», p. XXI.

28. G. Tarde, Les lois de l'imitation, 2001, p. 128.

29. Ibid., p. 203. 
L'absence d'imitation actuelle, quand elle est constatée, signifie plutôt, en réalité, sa cristallisation dans des ressemblances qui en sont comme le vestige. C'est toujours ensemble que l'imitation et l'invention constituent la dynamique d'engendrement de la société, car toute seule l'invention marquerait aussi bien une sortie en dehors du social: «Pour innover, pour découvrir [...], l'individu doit échapper momentanément à la société. Il est supra-social, plutôt que social, en ayant cette audace si rare ${ }^{30}$. Mais dès lors qu'elle est prolongée par ses reprises imitatives, l'invention retombe dans le social.

Or, lorsque Bergson mentionne l'imitation dans le premier chapitre des Deux sources, c'est au contraire pour qualifier une forme de relation supra-sociale entre les hommes, celle qui concerne la "morale ouverte", qu'il oppose à la «morale close», comprise comme la somme des obligations dont la fonction est d'assurer la stabilité et la conservation de l'ordre social. Le contenu de la morale des grands hommes de bien n'est pas étroitement social, et il y a en réalité deux manières de se sentir obligé. L'une qui provient d'une pression sociale anonyme d'origine biologique, c'est la tendance à se conserver en formant un groupe, et à obéir à un ensemble de règles qui assurent la cohésion de ce groupe par opposition à tout autre groupe. La seconde manière de se sentir obligé consiste en une aspiration en direction d'un idéal, qui est toujours incarnée par un individu, obligation qui garde une signification vitale, mais dans le sens cette fois d'une création, et qui s'adresse non pas à un groupe social déterminé, mais individuellement à tous les hommes. C'est alors sous la forme non d'une imitation, mais d'une émotion que cette obligation nous affecte et nous adresse son appel: «L'efficacité de l'appel tient à la puissance de l'émotion qui fut jadis provoquée, qui l'est encore ou qui pourrait l'être $[\ldots] »^{31}$.

À ce stade, on serait d'abord tenté de distinguer entre l'imitation dont le champ d'action serait étroitement social, et l'émotion qui serait suprasociale. Il n'en est rien. Chez Tarde aussi le champ de l'imitativité dépasse la socialité. Dans un passage des Lois sociales, où il répond à une objection, Tarde soutient en effet que l'imitation travaille à la constitution d'une société plus grande, d'une socialité élargie, plus vaste, au-delà des sociétés actuelles, et cela au-delà même des phénomènes de rivalité:

30. G. Tarde, Les lois de l'imitation, 2001, p. 147.

31. H. Bergson, Les deux sources..., p. 85. Sur le sens de l'émotion chez Bergson, nous renvoyons à notre étude: A. Bouaniche, "La force qui transporte et qui soulève": l'émotion dans la philosophie de Bergson", in Les émotions, S. Roux (dir.), Paris, J. Vrin (Thema), 2009, p. 211-234. 
N'est-il pas visible que, dans la mesure où les peuples rivaux, où les peuples ennemis s'assimilent leurs institutions, ils tendent à se fusionner? Il est donc bien certain que, non seulement entre individus associés déjà, chaque acte nouveau d'imitation tend à conserver ou à fortifier le lien social, mais encore qu'entre individus non encore associés, elle prépare l'association de demain, c'est-à-dire tisse déjà par des fils invisibles ce qui deviendra un lien manifeste ${ }^{32}$.

C'est dire que l'imitation tardienne et l'émotion bergsonienne sont créatrices de relation; elles possèdent une même nature communicative ou diffusive, et agissent l'une et l'autre par la production d'ondes ou de rayonnements :

L'émotion créatrice qui soulevait ces âmes privilégiées, et qui était un débordement de vitalité, s'est répandue autour d'elle: enthousiastes, elles rayonnaient un enthousiasme qui ne s'est jamais complètement éteint et qui peut toujours retrouver sa flamme ${ }^{33}$.

Ce que partagent l'émotion et l'imitation, c'est ainsi une même structure de leur dynamisme: du dedans vers le dehors, du centre vers la périphérie. Cette loi signifie pour Tarde que ce qui est imité n'est pas le plus superficiel, ni le plus extérieur, mais bien au contraire, et à chaque fois, le plus profond, le plus intérieur, à savoir la volonté et le sentiment, et non pas la simple attitude. Comme pour Bergson, l'affectivité est, pour Tarde, première sur l'idée, l'émotion sur la conception, la foi sur le dogme. De même pour Bergson, l'émotion est par essence un phénomène trans-subjectif d'entre-pénétration psychique, elle touche à l'intériorité. Ainsi l'émotion, ici paradigmatique, suscitée par une grande œuvre dramatique, est d'une autre nature que l'émotion passagère, superficielle, infra-intellectuelle, son processus n'est pas physique ou corporel, mais psychologique et même inter-psychologique: " unique en son genre, [l'émotion] a surgi dans l'âme du poète, et là seulement, avant d'ébranler la nôtre » ${ }^{34}$. Et lorsque Bergson évoque l' "atmosphère d'émotion » nécessaire aux grandes transformations socio-historiques, c'est en précisant aussitôt que, en sens inverse, lorsqu'elle gagne d'autres individus, l'émotion doit être assimilée, intériorisée, ingérée même, et que ce n'est qu'à cette condition qu'elle est effective, ce que Bergson suggère, en un passage, à travers une gradation de l'extérieur vers l'intérieur: «Si l'atmosphère d'émotion est là, si je l'ai respirée, si l'émotion me pénètre, j'agirai selon elle, soulevé par elle» ${ }^{35}$.

32. G. Tarde, Les lois sociales, p. 61.

33. H. Bergson, Les deux sources..., p. 97-98.

34. Ibid., p. 44.

35. Ibid., p. 45. Nous soulignons. 


\section{Un «principe de Huygens spirituel»}

Cette propagation du nouveau à partir d'une invention s'effectue à la faveur d'une véritable loi de diffusion qui n'est pas sans rappeler le principe de Huygens, d'après lequel tout point gagné par une onde originaire agit luimême comme une source d'ondes secondaire, qui réémet par conséquent, à son tour, un nouveau front d'ondes en direction de son voisinage, et ainsi de suite, indéfiniment; mais dès lors que cette diffusion s'opère dans le domaine psychique, c'est d'un véritable «principe de Huygens spirituel ${ }^{36}$ qu'il faudrait parler.

Lorsqu'il cherche à décrire ce mode de «diffusion rayonnante», Tarde le fait parfois en des termes qui ne sont pas sans évoquer un tel principe, comme par exemple dans Les lois de l'imitation: "Une pierre tombe dans l'eau, et la première onde produite se répète jusqu'aux limites du bassin ${ }^{37}$. Bergson a manifestement été frappé par ce passage, puisqu'il le reprend presque textuellement dans son discours de 1909, où il évoque ainsi la «leçon» de l'œuvre de Tarde:

En nous montrant comment la moindre de nos initiatives peut s'épanouir en conséquences incalculables, comment un simple geste individuel, tombant dans le milieu social comme une pierre dans l'eau d'un bassin, l'ébranle tout entier par des ondes imitatives qui vont toujours s'élargissant, elle nous donne un sentiment aigu de notre responsabilitée ${ }^{8}$.

Lorsqu'il évoque pour la première fois dans son œuvre, la force exercée par les grandes personnalités morales sur les autres hommes, Bergson le fait en des termes très proches, qu'il est difficile de ne pas rapporter à cette source tardienne:

Mais créateur par excellence est celui dont l'action, intense elle-même, est capable d'intensifier aussi l'action des autres hommes, et d'allumer généreuse, des foyers de générosité ${ }^{39}$.

La distinction cruciale entre les deux penseurs parait d'abord résider en ceci: l'irradiation de l'initial ne semble concerner pour Bergson que l'action vertueuse, c'est-à-dire rare, tandis que chez Tarde, c'est la totalité des conduites humaines (langage, techniques, sentiments, idées, etc.) qui

36. Nous empruntons cette expression à E. Husserl, Cinq articles sur le renouveau, L. Joumier (éd. et trad.), Paris, J. Vrin, 2005, p. 71.

37. G. Tarde, Les lois de l'imitation, 2001, p. 77.

38. H. Bergson, Écrits philosophiques, p. 376.

39. H. Bergson, «La conscience et la vie», in L'énergie spirituelle, F. Worms (éd.), Paris, PUF (Quadrige), 2009, p. 25. 
se propagent à tout instant par ondes imitatives. Mais plus profondément, c'est peut-être le statut de la nouveauté qui départagerait les deux penseurs sur le point qui nous occupe. Chez Tarde, l'invention est structurellement liée à l'imitation, dans sa genèse, comme dans son devenir. Sans l'imitation, l'invention ne survivrait pas à son apparition: elle ne doit finalement son existence qu'à son rayonnement imitatif. Mais Tarde précise également, et curieusement, que l'invention se résout elle-même en imitations, de sorte qu'il place «l'imitation à la source de l'invention $»^{40}$ :

Toutes les inventions et toutes les découvertes, donc, étant des composés qui ont pour éléments des imitations antérieures [...], et tous ces composés, imités à leur tour, étant destinés à devenir les éléments de nouveaux composés plus complexes, il suit de là qu'il y a un arbre généalogique de ces initiatives réussies, un enchaînement non pas rigoureux, mais irréversible, de leur apparition $[\ldots]^{41}$.

Chez Bergson, il y a certes une cristallisation inévitable des créations morales individuelles dans des lois ou des énoncés (ceux des droits de l'homme par exemple); mais l'émotion initiale (chrétienne toujours selon Bergson) peut toujours être retrouvée, intacte, vivante, palpitante, et irréductible. Ainsi certains préceptes moraux peuvent apparaître comme la "cendre d'une émotion éteinte»; mais «remuons la cendre», et dès lors «nous trouverons des parties encore chaudes, et finalement jaillira l'étincelle ${ }^{42}$, à partir de laquelle, à nouveau, les formules ébranleront notre volonté. C'est dire que la nouveauté morale peut à tout moment être retrouvée et nous affecter comme telle. C'est pourquoi Bergson parle de "continuation" plutôt que d'imitation: dans la continuation, la nouveauté "dure», se poursuit, "retentit» au lieu de simplement se prolonger, ce qui signifie que la garantie nous est toujours offerte de pouvoir à tout moment la réactiver ${ }^{43}$.

Il resterait à poser enfin la question de l'horizon ou du destin de cette propagation de l'imitation et de l'émotion, et ainsi, de la destination de l'humanité pour les deux auteurs. Tous deux voient finalement dans la démocratie le progrès politique essentiel dans l'histoire. Chez Tarde cependant, ce progrès ne fait qu'exprimer la reprise, dans le domaine social, d'une tendance universelle qui domine l'univers dans son intégralité, la tendance

40. B. Karsenti, «Présentation», p. XVIII.

41. G. Tarde, Les lois de l'imitation, 2001, p. 105; c'est Tarde qui souligne.

42. Pour cette formule et les précédentes, voir H. Bergson, Les deux sources..., p. 47.

43. Pour une distinction entre "prolongation» et «continuation», voir V. Jankélévitch, Traité des vertus, Paris, Flammarion (Champs essais), t. III, L’innocence et la méchanceté, 1986, p. 335 sq. 
à la similitude et au nivellement, à l'œuvre dans la nature physique aussi bien que vivante. Il écrit ainsi, qu'une fois admise la loi de rayonnement dans toutes les strates de la nature, on comprend que

la tendance à une assimilation cosmopolite et démocratique soit une pente inévitable de l'histoire, par la même raison que le peuplement uniforme et complet du globe et la calorification uniforme et complète de l'espace sont dans les vœux de l'Univers vivant et de l'Univers physique ${ }^{44}$.

Aux yeux de Bergson, la démocratie marque au contraire une rupture avec la nature, et avec les tendances de l'espèce. L'affirmation démocratique de la paix et de la valeur absolue de la personne humaine est une conquête historique qui vient rompre avec les tendances naturelles à la guerre et au nationalisme, inscrites dans la nature de l'homme. Mais pour Bergson, comme pour Tarde, le but final de l'histoire n'est pas dans un idéal politique et collectif. La démocratie ne vaut pour les deux penseurs que comme contexte d'actualisation de la "singularité profonde» des personnes. Seulement pour Tarde, cette actualisation, comme il l'indique dans les toutes dernières lignes des Lois de l'imitation, ne s'effectuerait que dans la «vie esthétique», conçue comme le "couronnement» de la «vie sociale». Pour Bergson, elle suppose un saut éthique au-delà de toute société constituée, en direction de l'humanité intégrale, où l'universel ne serait pas atteint «malgré», mais «à travers» la singularité, conformément à ce que Bergson considère, à la toute fin des Deux sources, comme «la fonction essentielle de l'univers» : « une machine à faire des dieux».

Arnaud Bouaniche

UMR 8163 - «Savoirs, textes, langage» / Centre international d'étude de la philosophie française contemporaine (CIEPFC) - ENS Ulm Professeur de philosophie en classes préparatoires, Lille

44. G. Tarde, Les lois de l'imitation, 2001, p. 439. 


\section{Annexe}

\section{Conférence de Bergson du 6 décembre 1904 (extrait)}

Ce passage est extrait de la séance d'un cours de Bergson donné au Collège de France et consacré à la question de la liberté. À cette occasion, et en ouverture de son propos, Bergson évoque brièvement l'œuvre de Tarde. Au-delà de l'hommage formel qu'il commence par rendre à celui qui l'a précédé dans l'occupation de sa chaire, il s'agit d'un précieux témoignage sur la place que Bergson accorde à Tarde dans sa pensée. En le rapprochant de Spencer, dont la doctrine évolutionniste est si déterminante dans son cuvre, Bergson reconnaît au sociologue une envergure inattendue. La manière par laquelle Bergson se rapporte aux deux auteurs dans son cuvre est cependant très différente: explicite et critique du côté de Spencer, elle est implicite et positive du côté de Tarde. Bergson leur reconnaît toutefois, à la fin de cet extrait, un itinéraire commun, d'une conception du monde à une conception de la société, celui en quelque sorte inverse qui le conduira de L'évolution créatrice aux Deux sources de la morale et de la religion, son dernier livre.

\section{Messieurs,}

Je devrais consacrer d'abord cette première leçon à l'œuvre du philosophe qui a occupé pendant malheureusement trop peu d'années la chaire où je me trouve transféré, philosophe dont nous conservons pieusement le souvenir, M. Gabriel Tarde.

Je me suis aperçu en relisant son œuvre qu'il est difficile, pour ne pas dire impossible, de la résumer en une leçon unique, et que cette œuvre qui est dominée par des idées et des vues de la plus haute importance, est peut-être plus importante encore par les vues de détail et par les suggestions de toutes sortes dont elle est pleine, car Tarde fut avant tout un grand évocateur d'idées. Il m’a semblé qu'il valait mieux consacrer à l'étude de cette œuvre non pas une leçon cette année, mais l'an prochain ou quelqu'une des années ultérieures, probablement l'année prochaine, un des cours de l'un des semestres, le cours du samedi, celui que nous allons consacrer cette année même au commentaire d'une œuvre de Herbert Spencer. Nous ferons pour La loi de l'imitation [sic], l'œuvre capitale de Tarde, ce que nous allons faire cette année pour Les premiers principes de Spencer, un commentaire critique.

Je rapproche ces deux noms l'un de l'autre, Tarde et Spencer, ce n'est pas sans raison. L'un des deux noms évoque l'autre; l'une des deux œuvres complète l'autre. Les conclusions de ces deux philosophes sont aussi opposées entre elles que des conclusions peuvent l'être; sur presque tous les points on pourrait opposer Tarde à Spencer, comme aussi le tempérament intellectuel, si on peut dire, le tempérament intellectuel de ces deux philosophes diffère profondément. Spencer, comme nous le verrons, est un déductif, un grand déductif et avant tout un déductif, malgré la masse énorme de faits dont son œuvre est pleine, mais l'examen approfondi de cette œuvre, comme d'ailleurs l'étude de sa biographie, montrent bien que ce fut 
avant tout un grand constructeur de systèmes. Il avait débuté comme ingénieur mécanicien et il resta constructeur toute sa vie, avec je ne sais quoi de mécanique dans sa manière de procéder. Tarde au contraire fut un intuitif, un grand intuitif à la différence de Spencer, qui, comme nous le verrons, a formulé un programme en 1860, programme où se trouvaient déjà indiqués, chapitre par chapitre, tous les livres qu'il devait faire, et qui a rempli son programme, qui a mis quarante ans à exécuter le programme tel qu'il l'avait conçu, marchant toujours droit devant lui.

Tarde au contraire alla de côté et d'autre, se promenant à travers les idées, se promenant pour son plaisir, semble-t-il, pour son plaisir à lui et pour le plaisir des autres. Il avait débuté, lui, dans la poésie; il avait commencé par faire des vers et on peut dire qu'il est resté poète toute sa vie. Comme tempérament intellectuel et au point de vue des conclusions où ils aboutissent, Tarde et Spencer sont très loin l'un de l'autre, cependant il y a une parenté entre eux, et cette parenté tient à deux causes: l'objet des deux philosophies est le même; Tarde, comme Spencer, est parti de la considération des sociétés; il a observé certaines conditions fondamentales de l'existence des sociétés et ce qu'il avait observé sur les sociétés il l'a étendu par voie de généralisation, de proche en proche, construisant ainsi un système du monde, dont le point de départ est sociologique. Voilà ce qu'a fait Tarde et voilà ce qu'a fait Spencer, et cette entreprise, ce me semble, est sans précédent dans l'histoire de la philosophie. Voilà le premier point. Puis il y a une autre ressemblance très profonde aussi, la ressemblance de méthode, en dépit de la diversité des conclusions, ressemblance d'autant plus frappante que les entreprises sont peu différentes l'une de l'autre et que pour atteindre ce résultat ils se sont servi avant tout du raisonnement par analogie. Tarde et Spencer sont les deux philosophes qui ont fait le plus grand usage, je peux dire le plus merveilleux usage, du raisonnement par analogie. Donc et par l'objet et par la méthode ils se ressemblent. Voilà pourquoi je disais que l'un de ces deux noms évoque l'autre; voilà pourquoi j'ajoute que l'étude de l'un de ces philosophes appelle l'étude de l'autre et que sans doute aussi l'historien de la philosophie plus tard les mettra très près l'un de l'autre. 